\title{
Abiotrophia Endocarditis in Children with No Underlying Heart Disease: A Rare but a Virulent Organism
}

\author{
Deepti P. Bhat, MD, ${ }^{*}$ Lakshmi Nagaraju, MD, ${ }^{\dagger}$ Basim I. Asmar, MD, ${ }^{\dagger}$ and Sanjeev Aggarwal, $\mathrm{MD}^{*}$ \\ Divisions of *Pediatric Cardiology and'Infectious Disease, The Carman and Adams Department of Pediatrics, Children's \\ Hospital of Michigan, Wayne State University School of Medicine, Detroit, Mich, USA
}

\begin{abstract}
A B S T R A C T
Infective endocarditis is extremely rare in children with structurally normal hearts. The most common etiological agents are staphylococcal and streptococcal species. Nutritionally variant streptococci also classified as Abiotrophia species are a group of fastidious organisms that account for only $5 \%$ to $6 \%$ of all cases of culture-negative infective endocarditis. Only seven cases of Abiotrophia infective endocarditis have been previously reported in children with no underlying structural heart disease. We report two cases of Abiotrophia infective endocarditis in children without any predisposing factors. Both patients presented with nonspecific symptoms leading to delay in diagnosis. While bacteriological clearance was achieved in both cases, both had a complicated course including development of brain mycotic aneurysms, splenic infarction, renal failure, and irreversible damage to the mitral valve. Both patients required surgical removal of the native mitral valve and replacement. We also present review of seven cases with similar diagnosis published previously in literature and highlight important differences. Our cases highlight special challenges in management of Abiotrophia endocarditis in pediatric patients. As the organism may not be isolated in routine culture media, may present with atypical clinical symptoms and may have a complicated course even without antibiotic failure, a high index of suspicion should be maintained in children with subacute symptoms even with no underlying structural cardiac disease.
\end{abstract}

Key Words. Nutritionally Variant Streptococci; Endocarditis; Children

\section{Introduction}

$\mathrm{I}$ nfective endocarditis (IE) is rare in children with an estimated incidence of 1 in 1280 admissions per year. ${ }^{1}$ Most cases of IE occur in patients with structural congenital or acquired heart disease (such as rheumatic heart disease) or in the setting of indwelling central catheters. ${ }^{2}$ IE is very rare in children with no underlying heart disease. Rosenthal et al. studied 85 pediatric cases of IE at a single center. Of these, only five patients $(6 \%)$ had no known underlying heart disease or other predisposing factors like indwelling catheters. ${ }^{2}$ Most cases of IE in these patients with normal hearts are attributed to Staphylococcus aureus and Streptococcus viridians. ${ }^{3-5}$ The term "culture-negative IE" is used for the cases when no organism is isolated and is seen in $5-7 \%$ of cases of IE., ${ }^{3,6,7}$ The reason for this has been ascribed to inappropriate culture techniques, slow-growing organisms, or organisms that are difficult to grow in regular culture media. ${ }^{8}$ Abiotrophia species previously classified as nutritionally variant streptococci (NVS) is one such fastidious organism that has been reported as a rare

Congenit Heart Dis. 2014;9:E116-E120 cause (5-6\% of all cases) of culture-negative IE. ${ }^{9-12}$ This organism has been reported to be associated with high relapse rates and treatment failures in adults., ${ }^{911}$ The infection is rare in children with only 17 reported cases in the worldwide literature. ${ }^{10,13}$ Of these, only seven have been reported in children with no underlying structural heart disease.${ }^{11-16} \mathrm{We}$ report two cases of IE in children with structurally normal hearts caused by Abiotrophia species and their outcomes and review of other published cases.

\section{Case Report 1}

An 11-year-old previously healthy boy was admitted with a 5-month history of recurrent migratory arthralgia. His initial symptoms were right ankle pain, which transiently improved with steroids followed by pain in the joints of hands and feet. The joint symptoms were preceded by streptococcal throat infection which was treated with two courses of antibiotics. There was no associated history of fever, rash, chest pain, exertional dyspnea, or syncope. The medical history was 
insignificant for any cardiac, dental, or systemic disease requiring indwelling central catheters.

On initial examination, he was afebrile (temperature $36.4^{\circ} \mathrm{C}$ ) with tachycardia (heart rate $123 /$ minute), mildly elevated blood pressure (124/ $79 \mathrm{~mm} \mathrm{Hg}$ ), and saturations of $99 \%$ on room air. Positive findings on examination included mild edema and redness of the right ankle joint and a grade 3/6 holosystolic murmur which was heard best in the axilla. His initial laboratory evaluation showed elevated C-reactive protein $(90 \mathrm{mg} / \mathrm{L})$, erythrocyte sedimentation rate ( $42 \mathrm{~mm} /$ hour), and rheumatoid factor $(156 \mathrm{IU} / \mathrm{mL})$. He had normal levels of anti-streptococcal DNAase titers (1:480), anti-streptococcal $\mathrm{O}$ titers $(141 \mathrm{IU} / \mathrm{mL})$, immunoglobulins, and complements. Magnetic resonance imaging of the lower limb joints showed no effusions or bony lesions. An echocardiogram showed moderate mitral insufficiency, no mitral valve stenosis with normal Doppler velocities in all other valves. No obvious vegetations were visualized on any valves. The biventricular function was normal with no evidence of pericardial effusion. A provisional diagnosis of IE was made and he was started on vancomycin and ceftriaxone.

The blood cultures drawn before the start of antibiotics remained negative for 3 days and then showed growth of the rare Abiotrophia species. Drug sensitivity patterns could not be assessed on this pathogen due to its fastidious growth requirements. He was then switched to ampicillin and gentamicin as empiric therapy for NVS as recommended by the infectious disease team. He underwent a repeat echocardiogram on day 7 , which now showed a $9 \mathrm{~mm} \times 9 \mathrm{~mm}$ vegetation on the superior surface of both the leaflets of mitral valve with moderate mitral regurgitation. Blood culture showed no growth after day 7 of treatment. Patient remained asymptomatic from the cardiovascular standpoint and was discharged home after 14 days on home intravenous antibiotics.

A week after discharge, he presented with highgrade fever $\left(39.5^{\circ} \mathrm{C}\right)$ and generalized headaches. A magnetic resonance imaging scan of the brain revealed a $5.5-\mathrm{mm}$ aneurysm involving the anterior opercular branch of the left middle cerebral artery with evidence of adjacent vasculitis (Figure 1). The aneurysms were managed conservatively with observation. Vancomycin was added in place of ampicillin. The repeat serial blood cultures showed no growth. His fever and headache resolved over the next 2 days and he was discharged home on vancomycin and gentamicin. Three days later, patient returned with complaints

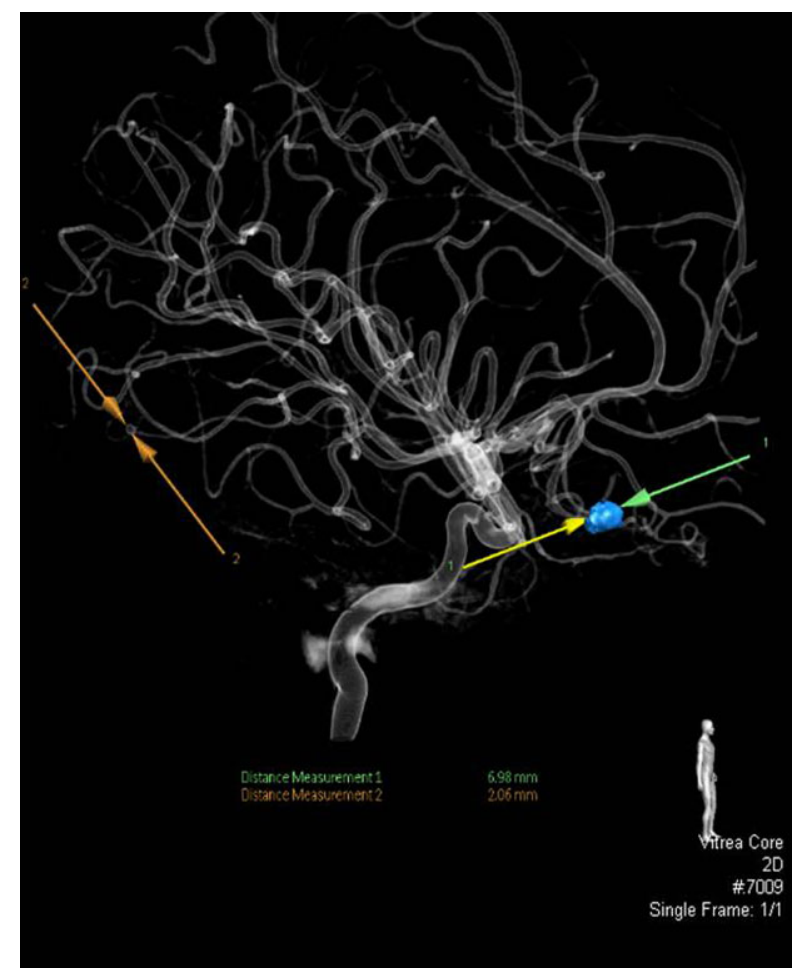

Figure 1. Magnetic resonance image of the brain in the first patient shows mycotic aneurysm in the left middle cerebral artery.

of severe left upper quadrant abdominal pain. An urgent abdominal computed tomography with contrast showed an infarcted area within the anterior spleen. He had no associated hemodynamic instability and was managed conservatively. At this time, his laboratory tests demonstrated acute deterioration of renal function with increase in creatinine $(9 \mathrm{mg} / \mathrm{dL})$ with hyponatremia (sodium: $129 \mathrm{meq} / \mathrm{L})$. He was diagnosed with nonoliguric renal failure which was thought to be related to a combination of endocarditis, nephro-toxic drugs, and intravenous contrast. Gentamicin was discontinued and he was continued on vancomycin monotherapy. His blood cultures remained negative. His renal function was monitored closely and gradually normalized.

His follow-up echocardiogram after completing 6 weeks of antibiotics showed no change in size of vegetations or severity of mitral valve regurgitation. Considering the high risk for intracranial bleeding, aneurysm rupture, or embolization, surgical replacement of the mitral valve was withheld. He subsequently underwent coil occlusion of his brain aneurysm. At 10 months follow-up, his echocardiogram showed significant mitral valve regurgitation, and he was therefore referred for 


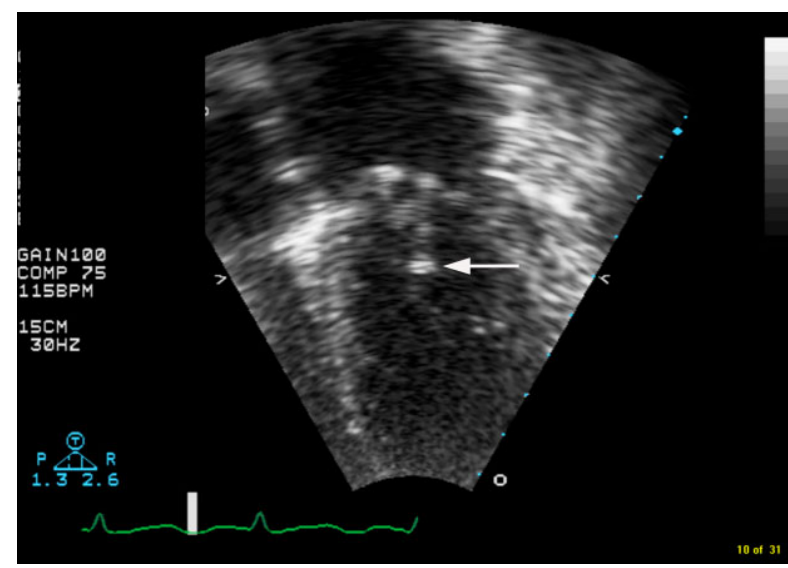

Figure 2. Transthoracic echocardiogram performed on the second patient shows thread-like vegetation on the anterior mitral valve leaflet.

surgery. During the surgery, he was found to have diffusely damaged valve leaflets and therefore underwent mechanical mitral valve replacement. He was clinically doing well at 6 months follow up after surgery.

\section{Case Report 2}

A 14-year-old previously healthy girl was admitted with 1-year history of recurrent cough, myalgia, and arthralgia. Her symptoms were preceded by removal of orthodontic braces. These episodes had been treated with several short courses of antibiotics and analgesics.

On initial examination, she was afebrile (temperature $37.3^{\circ} \mathrm{C}$ ) with normal heart rate ( 88 beats/ minute), blood pressure $(97 / 58 \mathrm{~mm} \mathrm{Hg})$, and saturations (98\% room air). Positive findings on examination included digital clubbing and a medium-pitched grade 3/6 holosystolic murmur which was best heard in the mitral area with radiation to the axilla. Her initial laboratory workup showed mild anemia (hemoglobin $11.6 \mathrm{gm} / \mathrm{dL}$ ), elevated erythrocyte sedimentation rate $(16 \mathrm{~mm} /$ hour), and rheumatoid factor $(8.9 \mathrm{IU} / \mathrm{mL})$. An echocardiogram revealed a long, thread-like vegetation on the anterior mitral leaflet with moderate to severe mitral valve insufficiency (Figure 2). All other valves were normal. The left atrium and ventricle were mildly dilated. The biventricular function was normal with no evidence of pericardial effusion.

She was empirically started on ampicillin and gentamicin as treatment for IE. Her blood culture showed growth of Abiotrophia species after 3 days of incubation which was found to be resistant to ceftriaxone but sensitive to penicillin, meropenem, and vancomycin. She was continued on the same antibiotics. The first five blood cultures drawn on the first day were positive, and subsequently, she had serial negative blood cultures. On day 10, she developed fever when she was switched to vancomycin; however, her repeat blood culture did not show any growth. She was discharged home after 17 days with a planned 6-week course of intravenous antibiotics.

Her follow-up echocardiogram after 4 weeks of treatment showed a decrease in the size of the vegetation while she continued to have moderate to severe mitral insufficiency. About 4 months after completing her antibiotic treatment, she was referred for surgery. During surgery, the anterior leaflet of the mitral valve was noted to be severely damaged, and the posterior leaflet was half the size of normal. A mitral valve replacement with mechanical valve was performed. The postoperative course was complicated by development of complete heart block which required placement of a permanent pacemaker. She was discharged home after 10 days. She was clinically doing well at 6 months follow up after surgery.

\section{Discussion}

IE is rare in children without underlying heart disease. ${ }^{2}$ Rosenthal et al. studied 85 pediatric cases of IE at a single center. Of these, only five patients $(6 \%)$ had no known underlying heart disease or other predisposing factors like indwelling catheters. ${ }^{2}$ Most cases of IE in these patients with normal hearts are attributed to S. aureus and S. viridians. ${ }^{3-5}$ IE caused by Abiotrophia species is extremely rare in children, with only 17 reported cases in the worldwide literature. ${ }^{10,13,17}$ Of these, nine had a structural heart disease and one was a neutropenic patient with long-term indwelling catheters. ${ }^{17}$ So far, only seven cases of Abiotrophia endocarditis have been reported in children with no underlying structural heart disease (Table 1). ${ }^{11-16}$ We report two pediatric cases of Abiotrophia endocarditis which highlight some important distinctive features of this rare infection. Both our patients were previously healthy with no underlying heart disease or predisposing factors such as central catheters. Both presented with atypical symptoms including migratory arthralgia, nonspecific fatigue, and absence of fever. The organism showed delayed growth, and drug sensitivity patterns could not be assessed due 
Table 1. Review of Seven Cases of Abiotrophia Endocarditis Reported in Children with No Known Underlying Structural Heart Disease or Indwelling Catheter

\begin{tabular}{|c|c|c|c|c|}
\hline Serial Number & Author, Year & Age (Years)/Sex & Course and Complications & Treatment \\
\hline 1 & Cayeux et al., $1971^{15}$ & $14 / \mathrm{M}$ & Mitral regurgitation & Surgical valve replacement \\
\hline 2 & Roberts and Sidlak, $1979^{12}$ & $13 / F$ & No relapse & Medical \\
\hline 3 & Feder et al., $1980^{16}$ & $7 / \mathrm{M}$ & Relapse & Medical \\
\hline 4 & Feder et al., $1980^{16}$ & $16 / \mathrm{F}$ & Relapse & Medical \\
\hline 5 & Handrick et al., $1988^{14}$ & $11 / \mathrm{F}$ & No relapse & Medical \\
\hline 6 & Raff et al., $2004^{13}$ & $5 / M$ & Aortitis, cardiac arrest & Surgical valve replacement \\
\hline \multirow[t]{3}{*}{7} & Lin and Hsu, $2007^{11}$ & $12 / \mathrm{F}$ & No relapse at 68 months & Medical \\
\hline & Present case 1 & $11 / \mathrm{M}$ & $\begin{array}{l}\text { Brain mycotic aneurysm, splenic infarction, } \\
\text { glomerulonephritis }\end{array}$ & Surgical valve replacement \\
\hline & Present case 2 & $14 / F$ & Severe mitral regurgitation & Surgical valve replacement \\
\hline
\end{tabular}

to its fastidious growth requirements. Importantly, bacterial clearance was achieved in both patients within a week of appropriate treatment. Nevertheless, one of them had a complicated course with embolic brain aneurysms and splenic infarct. Mitral valve function was severely affected in both patients, necessitating surgical replacement and need for lifelong anticoagulation.

Abiotrophia species belong to the genus of NVS which are a group of fastidious nonhemolytic streptococci that were first isolated in 1961 by Frenkel and Hirsch from patients with endocarditis and otitis media. ${ }^{18}$ The NVS are estimated to be responsible for about $5 \%$ to $6 \%$ of cases of culture-negative endocarditis. ${ }^{12,19,20}$ These pathogens are called "nutritionally variant" because they require addition of $0.01 \% \mathrm{~L}$-cysteine or $0.001 \%$ pyridoxal to ordinary culture media for their growth. ${ }^{15}$ Bouvet et al. further classified the NVS into two species, Streptococcus adjacens and Streptococcus defectiva, based on DNA-DNA hybridization technique. ${ }^{21}$ These two species together constitute the genus Abiotrophia, which consists of the species Abiotrophia defective and Abiotrophia adiacens. ${ }^{22}$

Abiotrophia endocarditis has been rarely reported in children. Chang et al. reviewed 13 cases of IE caused by NVS in children reported in English literature since $1971 .^{10}$ Nine of these patients $(69 \%)$ had some underlying heart disease. We performed a review of the literature and found that only seven pediatric patients with no structural heart disease have been previously reported (Table 1). ${ }^{11-16}$ Five of these patients $(71 \%)$ were managed with antibiotics alone and only two (29\%) patients required surgical replacement of the valve due to severe mitral regurgitation and aortitis, respectively. This is in contrast to our two patients who both required surgical valve replacement, necessitating lifelong anticoagulation.

Several studies in adults have reported high rates of complications in Abiotrophia endocarditis including bacteriological failure rate up to $40 \%$, mortality rates of $17-20 \%$ from congestive cardiac failure, and embolization in up to $27 \%$ of cases. ${ }^{7,23,24}$ This has been attributed to difficulty in isolating this organism and high drug resistance rates. In contrast, both our patients achieved relatively rapid bacteriological clearance. Despite this, both our patients had complicated course, with our first patient developing embolic mycotic aneurysms in cerebral vessels. Only three cases of mycotic aneurysms have been previously reported secondary to Abiotrophia endocarditis. ${ }^{25-27}$ All three of these were adults and two had subarachnoid hemorrhages. To the authors' knowledge, our first patient is the first pediatric case of multiorgan embolization due to mitral valve Abiotrophia endocarditis without antibiotic bacteriological failure.

Our cases highlight special challenges in the management of Abiotrophia endocarditis in pediatric patients. Despite appropriate antibiotic therapy and achievement of bacteriological clearance, patients are at high risk for embolic and immunemediated complications and irreversible valve damage. These patients require close monitoring and early involvement of surgical team for management. As the organism may not be isolated in routine culture media, and may present with atypical clinical symptoms, a high index of suspicion should be maintained in children with subacute symptoms even with no underlying heart disease.

\section{Author Contribution}

DB acquired the data, drafted and revised the manuscript. $\mathrm{LN}$ acquired the data and helped in drafting the manuscript. BA revised the manuscript. SA drafted and revised the manuscript.

Corresponding Author: Deepti P. Bhat, MD, Division of Pediatric Cardiology, Children's Hospital of Michi- 
gan, 3901, Beubien Boulevard. Detroit, MI 482012119, USA. Tel: (+1) 3137455993; Fax: (+1) 3139930894; E-mail: drdeeptibhat@yahoo.com

Conflicts of interest: None.

Disclosures: None.

Accepted in final form: April 8, 2013.

\section{References}

1 Van Hare GF, Ben-Shachar G, Liebman J, Boxerbaum B, Reimenschneider TA. Infective endocarditis in infants and children during the past 10 years: a decade of change. Am Heart 7. 1984;107:1235-1240.

2 Rosenthal LB, Feja KN, Levasseur SM, Alba LR, Gersony W, Saiman L. The changing epidemiology of pediatric endocarditis at a children's hospital over seven decades. Pediatr Cardiol. 2010;31:813-820.

3 Johnson CM, Rhodes KH. Pediatric endocarditis. Mayo Clin Proc. 1982;57:86-94.

4 Wei HH, Wu KG, Sy LB, Chen CJ, Tang RB. Infectious endocarditis in pediatric patients: analysis of 19 cases presenting at a medical center. 7 Microbiol Immunol Infect. 2010;43:430-437.

5 Saxena A, Aggarwal N, Gupta P, Juneja R, Kothari SS, Math R. Predictors of embolic events in pediatric infective endocarditis. Indian Heart 7. 2009;61: 242-245.

6 Martin JM, Neches WH, Wald ER. Infective endocarditis: 35 years of experience at a children's hospital. Clin Infect Dis. 1997;24:669-675.

7 Stockheim JA, Chadwick EG, Kessler S, et al. Are the Duke criteria superior to the Beth Israel criteria for the diagnosis of infective endocarditis in children? Clin Infect Dis. 1998;27:1451-1456.

8 Fukushige J, Igarashi H, Ueda K. Spectrum of infective endocarditis during infancy and childhood: 20-year review. Pediatr Cardiol. 1994;15:127-131.

9 Sharaf MA, Shaikh N. Abiotrophia endocarditis: case report and review of the literature. Can 7 Cardiol. 2005;21:1309-1311.

10 Chang HH, Lu CY, Hsueh PR, Wu MH, Wang JK, Huang LM. Endocarditis caused by Abiotrophia defectiva in children. Pediatr Infect Dis 7. 2002;21: 697-700.

11 Lin CH, Hsu RB. Infective endocarditis caused by nutritionally variant streptococci. Am $7 \mathrm{Med}$ Sci. 2007;334:235-239.

12 Roberts KB, Sidlak MJ. Satellite streptococci. A major cause of "negative" blood cultures in bacterial endocarditis? FAMA. 1979;241:2293-2294.

13 Raff GW, Gray BM, Torres A Jr, Hasselman TE.
Aortitis in a child with Abiotrophia defectiva endocarditis. Pediatr Infect Dis 7. 2004;23:574-576.

14 Handrick W, Kohler W, Spencker FB, Schneider P. Endocarditis due to nutritionally variant streptococci. Infection. 1988;16:371-372.

15 Cayeux P, Acar JF, Chabbert YA. Bacterial persistence in streptococcal endocarditis due to thiolrequiring mutants. 7 Infect Dis. 1971;124:247-254.

16 Feder HM Jr, Olsen N, McLaughlin JC, Bartlett $\mathrm{RC}$, Chameides L. Bacterial endocarditis caused by vitamin B6-dependent viridans group Streptococcus. Pediatrics. 1980;66:309-312.

17 Lopardo H, Mastroianni A, Casimir L. [Bacteremia due to Abiotrophia defectiva in a febrile neutropenic pediatric patient]. Rev Argent Microbiol. 2007;39:9394.

18 Frenkel A, Hirsch W. Spontaneous development of $\mathrm{L}$ forms of streptococci requiring secretions of other bacteria or sulphydryl compounds for normal growth. Nature. 1961;191:728-730.

19 Ruoff KL. Nutritionally variant streptococci. Clin Microbiol Rev. 1991;4:184-190.

20 Bottone EJ, Thomas CA, Lindquist D, Janda JM. Difficulties encountered in identification of a nutritionally deficient streptococcus on the basis of its failure to revert to streptococcal morphology. 7 Clin Microbiol. 1995;33:1022-1024.

21 Bouvet A, van de Rijn I, McCarty M. Nutritionally variant streptococci from patients with endocarditis: growth parameters in a semisynthetic medium and demonstration of a chromophore. 7 Bacteriol. 1981; 146:1075-1082.

22 Carey RB, Gross KC, Roberts RB. Vitamin B6-dependent Streptococcus mitior (mitis) isolated from patients with systemic infections. 7 Infect Dis. 1975;131:722-726.

23 Brouqui P, Raoult D. Endocarditis due to rare and fastidious bacteria. Clin Microbiol Rev. 2001;14:177207.

24 Stein DS, Nelson KE. Endocarditis due to nutritionally deficient streptococci: therapeutic dilemma. Rev Infect Dis. 1987;9:908-916.

25 Leonard MK, Pox CP, Stephens DS. Abiotrophia species bacteremia and a mycotic aneurysm in an intravenous drug abuser. N Engl 7 Med. 2001;344: 233-234.

26 Kohok DD, Parashar A, Punnam V, Tandar A. Subarachnoid hemorrhage in a patient with Abiotrophia defectiva endocarditis. Am $\mathcal{F}$ Med Sci. 2011;341:157159.

27 Yang YS, Shang ET, Lin JC, Chiu CH, Chang JY. A ruptured cerebral mycotic aneurysm caused by Abiotrophia defectiva endocarditis. Am $7 \mathrm{Med}$ Sci. 2010;339:190-191. 